Corresponding Author: Hammad Ali Fadlalmola; Tel: +966504900120 email:

hazzminno345@gmail.com

Received 5 May 2020

Accepted 24 June 2020

Published 30 June 2020

Production and Hosting by Knowledge E

(c) Hammad Ali Fadlalmola and Amal Mohamed

Elhusein. This article is

distributed under the terms of the Creative Commons

Attribution License, which

permits unrestricted use and

redistribution provided that

the original author and

source are credited.

Editor-in-Chief:

Prof. Mohammad A. M. Ibnouf
Research Article

\section{Nurse's Knowledge and Practice on the Care of Preterm Infants at Khartoum State Hospitals}

\author{
Hammad Ali Fadlalmola ${ }^{1}$ and Amal Mohamed Elhusein ${ }^{2}$ \\ ${ }^{1}$ Nursing College, Taibah University, Madinah, Saudi Arabia \\ ${ }^{2}$ Nursing Department, Bisha University, Bisha, Saudi Arabia
}

\section{Abstract}

Background: Premature infants can develop a range of problems because their immature organs. A proper nursing care for a premature baby should be established through good nursing performances. This study aimed to assess nurse's knowledge and practices regarding the care of a premature baby in a neonatal intensive care unit (NICU).

Methods: This study is a descriptive cross-sectional study, with a systematic collection, analysis, and interpretation of data that reports a clear picture of a particular situation carried out by 72 eligible nurses who were a graduate in BSc., and had at least six months' experience at the time of the study.

Result: The study showed that the percentage of nurse's knowledge regarding care of premature; definition of preterm neonate $44.4 \%$, nurses' had poor knowledge, umbilical vessels: Two arteries \& one vein $40.3 \%$, nurses' had poor knowledge, and the percentage of nurses' practices regarding care of premature baby; hand washing o $36.1 \%$, nurses' have poor practice, so there were poor nurses' knowledge and practices regarding care of premature baby.

Conclusion: Based on the results of our study, we concluded that most of the nurses had relatively poor knowledge and practices about the aspects of premature care.

Keywords: nurses' performances, preterm neonate, neonate in Intensive Care Unit, nursing care, Sudan

\section{Introduction}

TheWorld Health Organization (WHO) defines a preterm birth as all births before the completion of 37 weeks of gestation or $<259$ days since the last day of a woman's menstrual period [1]. Preterm birth has become a worldwide epidemic that is estimated at a global incidence of 15 million per year [2]. It is a major cause of death and a significant cause of long-term human loss worldwide. The complications of preterm birth are the 
million deaths per year and the second most common cause of under-five deaths after pneumonia [3]. An estimated number of 13 million babies are born premature annually. Rates are generally highest in low- and middle-income countries, and are on the rise in few middle- and high-income countries, particularly the Americas. Preterm birth is the leading direct cause of neonatal death (27\%); more than one million preterm newborns die annually [4]. Although, according to the $\mathrm{WHO}$, more than $60 \%$ of the preterm births occur in Africa and South Asia, it is truly a global problem. In the lower-income countries, on average, $12 \%$ of babies are born too early compared with the $9 \%$ in the higher-income countries. Within these countries, the poorer families are at a higher risk [5].

In a study by Lawn et al., it was reported that most premature babies (> 80\%) are born between 32 and 37 weeks of gestation, and many die needlessly due to the lack of simple care. The authors outline a series of packages of essential care for every newborn that are includes support for immediate and exclusive breastfeeding, thermal care, and hygienic cord, and skin care. For babies who do not breathe at birth, rapid neonatal resuscitation is crucial [6]. Innovative solutions are urgently need to prevent preterm birth worldwide, for example, there is a need of improvement in the antenatal, obstetric, and newborn cares to increase the survival rate [7]

Since there were not many studies on the topic of premature infants' nursing care, particularly focusing on nursing, it encouraged us to address this topic. . Two studies were conducted in Jordan and Iraq, but they were more focused on the respiratory distress syndrome of a newborn in general and not just preterm, they recommended on providing an educational program for nurses for improving the quality of nursing care of neonatal respiratory distress syndrome $[8,9]$.

In Sudan, where the current study was conducted, a good and meticulous search was performed in the literature, and no study matching the subject of the current study was found. Yet, there are some studies that dealt with some aspects related to the subject of the study. However, there is a reference to work that assesses the effectiveness of education program on nurses' knowledge and performance [10].

\subsection{Problem statement}

The investigators observed that the nurses had limited information regarding the care of premature babies. 


\subsection{Justification and rationale}

Previous studies about the care of preterm neonates have ignored the important nursing practices, for example the care of umbilical catheter, preterm care on continuous positive airways pressure (CPAP), ventilator, and incubator care.

A good nursing care of a preterm baby plays an important role in reducing morbidity and mortality and improve the survival for newborns.

\subsection{Research question}

What is the level of knowledge and practice of nurses about nursing care of a preterm infant?

\subsection{Objectives}

\subsubsection{General objective}

To assess nurse's knowledge and practices regarding the care of preterm infants in the neonatal intensive care unit (NICU) in Khartoum state hospitals.

\subsubsection{Specific objectives}

To assess nurses' knowledge about preterm care and compare their knowledge with their practices regarding the care of preterm neonates.

\section{Materials and Methods}

\subsection{Study design}

This is a descriptive cross-sectional hospital-based study, involving a systematic collection, analysis, and interpretation of data to give a clear picture of a particular situation.

\subsection{Study setting}

The NICU is distributed in the main two localities, (Khartoum and Omdurman)

This descriptive study was carried out in an NICU at the Jaafer Ibn Ouf Pediatrics Specialized Hospital, which is located in Khartoum State on the Mak nemir St. north of 
the dermatological hospital and consists of four rooms. The study was also carried out in the Omdurman Maternity Hospital, which is located in Omdurman state. The hospital contains one NICU with three rooms.

\subsection{Study population}

The focused groups of the study were the nurses working in the NICU. The selected nurses were BSc. graduates and had at least six months' experience.

\subsection{Inclusion criteria}

Nurses working in the NICUs who accepted to participate in the study, had a BSc. certificate and at least six months' of experience.

\subsection{Exclusion criteria}

Nurses who did not agree to participate in the study, were absent at the time of data collection time, and those that had less than six months of experience were excluded from the study.

\subsection{Sampling}

\subsubsection{Sample size and technique}

A total of 42 nurses from the Omdurman Maternity Hospital and 30 from the Jaafer Ibn Ouf Hospital participated in the study.

\subsection{Methods of data collection}

This descriptive cross-sectional study design involves a systematic collection, analysis, and interpretation of data to give a clear picture of a particular situation which was carried out on 72 eligible nurses qualified for the study and who were available during the study period. The study focused mainly on the nursing process such as the care of umbilical catheter, preterm care on CPAP, ventilator and incubator care, gavages feeding, oxygen administration, and care of a baby under phototherapy. 


\subsubsection{Data collection tools}

The data was obtained through interview questionnaires. Each participating nurse was provided a checklist to cross-assess their practice. Therefore, the data collection technique was administrated through written questionnaire and observation.

\subsubsection{Data analysis}

Data was entered and analyzed using the SPSS program version 20. It was organized, tabulated, and analyzed based on the objectives of the study using descriptive statistics, that is, the percentage, mean, and standard deviation, represented as figures and tables in the article.

\section{Results}

Of the 72 nurses participating in the study, about one-fifth (20.8\%) stated that they needed training courses on CPAP, and a minority (2.8\%) of them stated that they needed courses on the care of preterm infants. This indicates that the nurses had poor knowledge about the care of preterm infants as shown in Figure 1. Moreover, about $68.1 \%$ of the study samples stated that a preterm baby is a baby born before 24 weeks of gestation, while only $5.6 \%$ knew it refers to a baby born before 40 weeks of gestation, which indicates the nurses' low level of knowledge about the definition of preterm baby itself (Table 1). Additionally, about $86.1 \%$ of the nurses considered respiratory distress syndrome as a common complication of prematurity, and $61.1 \%$ of them stated neonatal septicemia as a common complication of prematurity (Table 2).

Regarding the susceptibility of the preterm infant to infection, 93.1\% of the responders stated that low immunity and $19.4 \%$ of them stated that anemia increases the susceptibility of preterm infant to infection as shown in Figure 2.

TABLE 1: Distribution of the study samples according to their knowledge regarding the definition of a preterm neonate $(N=72)$

Definition of preterm neonate
a. Baby born before 24 weeks of gestation
b. Baby born before 37 weeks of gestation
c. Baby born before 40 weeks of gestation
Total

\begin{tabular}{|c|c|}
\hline \multicolumn{2}{|c|}{ Yes } \\
\hline Frequency & Percentage \\
\hline 49 & $68.1 \%$ \\
\hline 32 & $44.4 \%$ \\
\hline 4 & $5.6 \%$ \\
\hline 72 & $100 \%$ \\
\hline
\end{tabular}




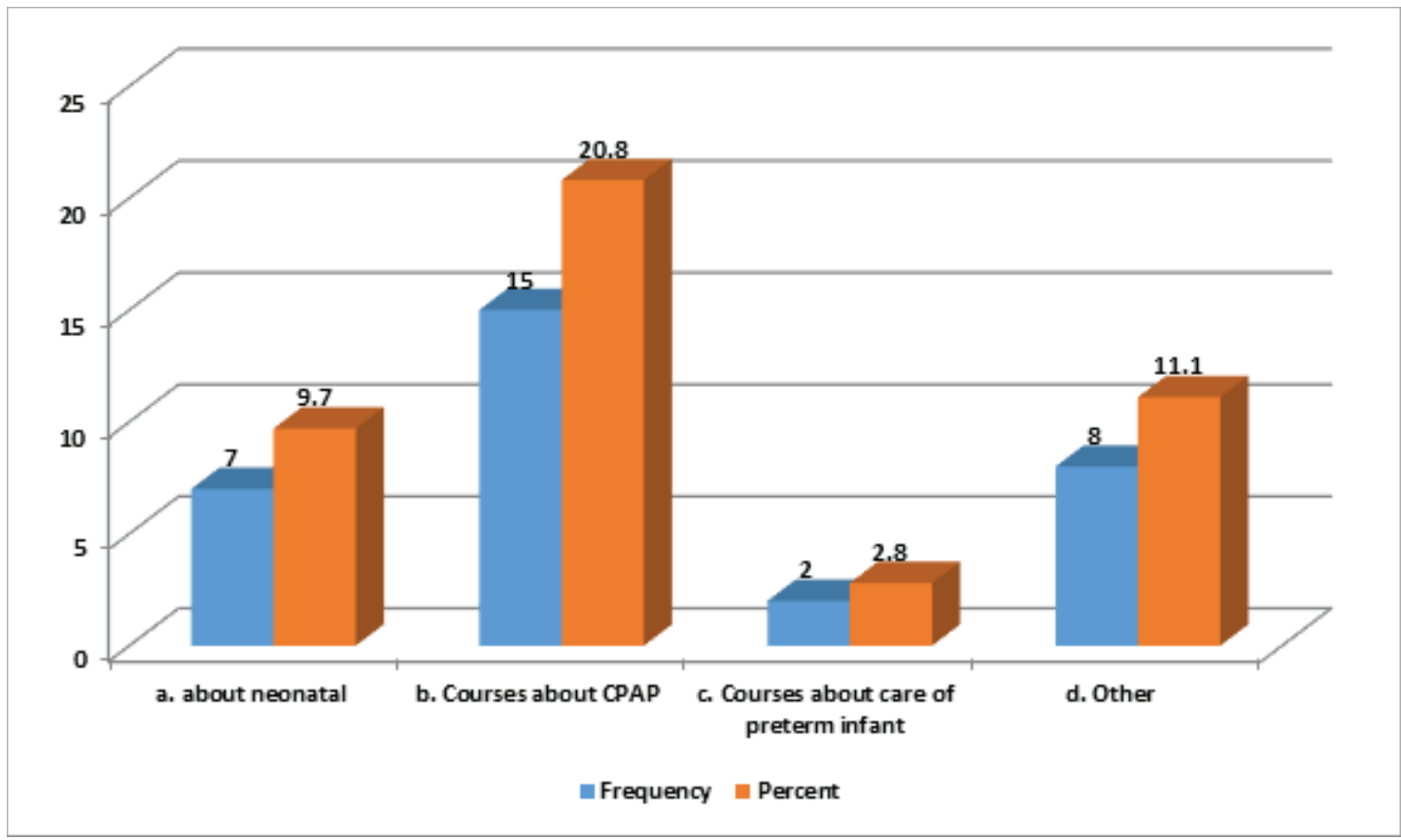

Figure 1: Distribution of the study samples according to their knowledge regarding the training courses.

TABLE 2: Distribution of the study samples according to their knowledge regarding the common complication of prematurity $(N=72)$

Common complication of prematurity
a. Respiratory distress syndrome
b. Anemia and hemorrhage
c. Necrotizing enterocolitis
d. Neonatal septicemia
Total

\begin{tabular}{|c|c|}
\hline \multicolumn{2}{|c|}{ Yes } \\
\hline Frequency & Percentage \\
\hline 62 & $86.1 \%$ \\
\hline 20 & $27.8 \%$ \\
\hline 21 & $29.2 \%$ \\
\hline 44 & $61.1 \%$ \\
\hline 72 & $100 \%$ \\
\hline
\end{tabular}

\section{Discussion}

The study shows that nurses' had poor knowledge and practices regarding the care of a premature baby.

As per the $\mathrm{WHO}$ scores concerning the knowledge, $0-50 \%$ is considered as poor, $50-75 \%$ as fair, and $(75-100 \%$ as[11].

About $43.1 \%$ of the study samples had an experience of six months that lead to the poor knowledge of the nurses, as supported by a previous study [12]. Of the participants who had at least one year of experience working with newborns, about $98.6 \%$ had a bachelor's degree in nursing sciences.

Further, about $20.8 \%$ of the study samples stated that they needed a training on CPAP, and only $2.8 \%$ thought they needed a training on the preterm infant care. 


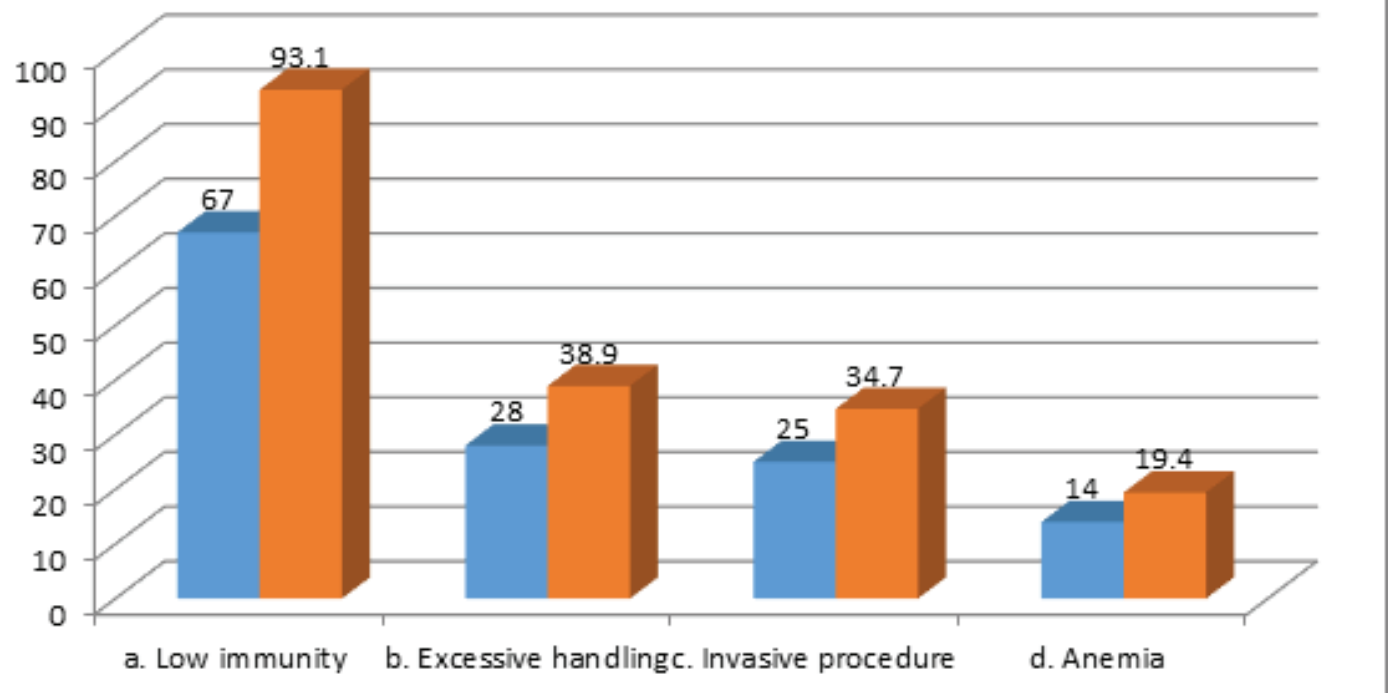

Figure 2: Distribution of the study samples according to their knowledge regarding the susceptibility of preterm infant to infection.

While $68.1 \%$ of the study samples stated that a preterm is baby born before 24 weeks of gestation, only $5.6 \% \mathrm{knew}$ that it refers to a baby born before 40 weeks' gestation.

The current result revealed that $86.1 \%$ of the nurses stated respiratory distress syndrome as a common complication of prematurity, which is considered as a good knowledge, and about $61.1 \%$ of them stated neonatal septicemia as a common complication, which was termed as a fair knowledge [11, 13].

About $93.1 \%$ of the responders stated that low immunity makes a preterm infant susceptible to infection, which was good knowledge in relation to a literature review, and only $19.4 \%$ said that anemia makes them susceptible to infection, which shows their poor knowledge on the subject.

With respect to complications, $91.7 \%$ of the study sample stated that infection is a complication of umbilical vein catheterization, showing nurses' good knowledge in relation to Prof Nicholas Evans' study [14] and 51.4\% considered Thromboembolism as a complication, showing a fair knowledge in relation to the literature review. About 38.9\% of the nurses stated that infection can be reduced by attaching three-way taps to the end of each umbilical venous catheters (UVC) lumen, showing their poor knowledge in relation to study which stated the attach 3 way taps to end of each UVC reduce the infection [15]. and a quarter (75.0\%) of them they stated that easy manipulation.

It was observed that the majority (80.6\%) of the study samples stated that the incubator is indicated for all preterm infant $<34$ weeks, showing the nurses' had good 
knowledge in relation to study which stated that an incubator is indicated for all preterm infant $<34$ weeks [16].

About $59.7 \%$ of the study samples stated that nurse infant supine or in lateral position for 24 hours post-procedure, third-fifth (62.5\%) of the study sample they said that doesn't cover the umbilical stump, the nurses' fair practice. Only $4.2 \%$ of the study samples stated that humidification of incubator should be started in all preterm with $<31$ weeks' gestation at $85 \%$ humidity, showing that the nurses' had a poor knowledge with respect to the study of Harpin and Rutter, which stated that humidification of incubator should be started in all preterm < 31 weeks' gestation at $85 \%$ humidity [17].

About $4.2 \%$ of the study samples said that handwashing is important for the preterm care while gavage feeding, showing the nurses' poor knowledge with respect to Abaker's study [18].

About $97.2 \%$ of the responders stated that gavage feeding tube should be checked and fixed with an adhesive tip, and $94.4 \%$ of them said that the correct position of the tube in the stomach should also be checked, showing that the nurses had a good practical knowledge of Quesenberry and Hillyers study, which list handwashing, measuring the placement of the tube from tip of the nose to ear lobe and then up to xiphoid, lubricating the tube with lubrication jelly, checking the correct position of the tube in the stomach, withdrawing injection air from the stomach and aspirating the gastric content, fixing the tube with adhesive tip, disposing waste material as per hospital policy as important factors for preterm care [19].

About $95.8 \%$ of the nurses said that the eye and genitalia should be covered during phototherapy, and about $84.7 \%$ stated that baby's temperature should be monitored frequently, showing that the nurses had a good knowledge with respect to Murphy et al.'s study. Additionally, only about $23.6 \%$ of the study sample stated that fluid balance should be monitored carefully, showing nurses' poor knowledge.

About $56.9 \%$ of the study samples knew about teaching mothers about breastfeeding, showing their fair knowledge of the WHO and UNICEF guidelines. Besides, only 13.9\% of them explained parent about the environmental hygiene, follow-up plan and immunization, reflecting their poor knowledge on teaching mothers about breastfeeding, explaining about environmental hygiene, and follow-up plan and immunization [20] .

\section{Conclusion}

Based on the results of the current study, we conclude that most nurses had relatively poor knowledge and practices about the aspects of premature care. They had adequate 
knowledge about gavages feeding tube and that it must be fixed with adhesive tip, but poor knowledge regarding the humidification of incubator, care of baby on CPAP, umbilical vessels, preparation, and procedure of UVC, teaching and supporting the parent.

\section{Acknowledgements}

The authors acknowledge the two hospitals in which conduct the research was conducted and thank all nurses that participated in the study.

\section{Ethical Considerations}

An official letter from the supervisor of the Faculty of Nursing Science, Khartoum University was issued to the manager of the Omdurman Maternity Hospital and the Jaafer Ibn Ouf Pediatric Hospital to carry out this study in their hospitals, ensuring confidentiality and preserving information. All nurses gave a written informed consent prior to the study.

\section{Competing Interests}

The authors declare that there was no conflict of interest.

\section{Funding}

This research did not receive any specific grant from any funding agency in the public, commercial, or not-for-profit sectors.

\section{References}

[1] Linked to "Born too Soon: The Global Action Report on Preterm Birth." Country data and rankings for preterm birth EMBARGO UNTIL MAY 2ND 2012. "Born too Soon Glob. Action Rep. Preterm Birth," 2012. Retrieved from: http://www.who.int/pmnch/ media/news/2012/201204_borntoosoon_countryranking.pdf

[2] Purisch, S. E. and Gyamfi-Bannerman, C. (2017). Epidemiology of preterm birth. Seminars in Perinatology. 
[3] Mondal, M. N. I., Hossain, M. K., and Ali, M. K. (2009). Factors influencing infant and child mortality: a case study of Rajshahi District, Bangladesh. The Journal of Human Ecology, vol. 26, no. 1, pp. 31-39.

[4] Lawn, J. E., Gravett, M. G., Nunes, T. M., et al. (2010). Global report on preterm birth and stillbirth (1 of 7): definitions, description of the burden and opportunities to improve data. BMC Pregnancy Childbirth, vol. 10, no. 1, p. S1.

[5] WHO. (2015). Preterm Birth fact sheet. Retrieved from: https://www.who.int/newsroom/fact-sheets/detail/preterm-birth

[6] Lawn, J. E., Davidge, R., Paul, V. K., et al. (2013). Born too soon: care for the preterm baby. Reproductive Health, vol. 10, no. 1, p. S5.

[7] Simmons, L. E., Rubens, C. E., Darmstadt, G. L., et al. (2010).Preventing preterm birth and neonatal mortality: exploring the epidemiology, causes, and interventions. Seminars in Perinatology, vol. 34, no. 6, pp. 408-415.

[8] Aziz, A. R. and Mansi, Q. H. (2017). Assessment quality of nursing care provided to neonates with respiratory distress syndrome at Intensive Care Unit in Al-Nasiriyah city hospitals. Kufa Journal for Nursing Sciences, vol. 7, no. 2, pp. 1-12.

[9] Ali, R. A., Obeisat, S. M., and Tarawneh, L. H. (2019).Improving nursing knowledge and care for neonates with respiratory distress in Jordan. International Nursing Review, vol. 66 , no. 3 , pp. 338-345.

[10] Fadlalmola, H. A. and Elkareem, E. M. A. (2020). Impact of an educational program on knowledge and quality of life among hemodialysis patients in Khartoum state. International Journal of Africa Nursing Sciences, vol. 12, p. 100205.

[11] Desgualdo, C. M., Riera, R., and Zucchi, P. (2011). Cost estimate of hospital stays for premature newborns in a public tertiary hospital in Brazil. Clinics, vol. 66, no. 10, pp. 1773-1777.

[12] You, L.-M., Aiken, L. H., Sloane, D. M. (2013). Hospital nursing, care quality, and patient satisfaction: cross-sectional surveys of nurses and patients in hospitals in China and Europe. International Journal of Nursing Studies, vol. 50, no. 2, pp. 154-161.

[13] Gomella, T. L., Cunningham, M. D., Eyal, F. G., et al. (2013). Neonatology: Management, Procedures, On-call Problems, Diseases, and Drugs. McGraw-Hill Education Medical.

[14] Health Sydney Local Health District. (2017). Provisional Fellow in Neonatal Medicine at RPAH. Retreived from: https://www.slhd.nsw.gov.au/rpa/neonatal/fellow.html

[15] Pack, N. L. L., Dressing, T., and Balls, C. (2014). Procedure for: umbilical venous catheterisation-(UVC). 
[16] Sauer, P. J., Dane, H. J., and Visser, H. K. (1984). New standards for neutral thermal environment of healthy very low birthweight infants in week one of life. Archives of Disease in Childhood, vol. 59, no. 1, pp. 18-22.

[17] Harpin, V. A. and Rutter, N. (1985). Humidification of incubators. Archives of Disease in Childhood, vol. 60, no. 3, pp. 219-224.

[18] Abaker, M. A. A. (2017). Nurses' Knowledge Regarding Nursing Care of Neonatal Hypothermia at Gaffer Ibnuf Pediatric Hospital, Khartoum State, Sudan (2017). Sudan: University of Gezira.

[19] Quesenberry, K. E. and Hillyer, E. V. (1994). Supportive care and emergency therapy. Avian Medicine: Principles and Applications, vol. 382, p. 416.

[20] Murphy, G. M., McCann, P., O'Leary, A., et al. (1997). Guidelines for the use of phototherapy and photochemotherapy in Ireland. Irish Journal of Medical Science, vol. 166, no. 2, p. 92. 